\title{
Preoperative transarterial embolization for solitary fibrous tumor of the tongue: A case report
}

\author{
ON HASEGAWA $^{1}$, MASATO WATANABE ${ }^{1}$, MICHIHIDE KONO $^{1}$, DAISUKE YUNAIYAMA ${ }^{2}$ and DAICHI CHIKAZU ${ }^{1}$ \\ Departments of ${ }^{1}$ Oral and Maxillofacial Surgery, ${ }^{2}$ Radiology, Tokyo Medical University, Shinjyuku-ku, Tokyo 160-0023, Japan
}

Received April 29, 2020; Accepted October 27, 2020

DOI: $10.3892 / \operatorname{mco} .2021 .2217$

\begin{abstract}
Solitary fibrous tumors (SFTs) are derived from mesenchymal cells originating mainly from the pleura. Reports of bleeding SFTs in head and neck regions are rare. A number of reports have focused on tongue SFT treatments, but to the best of our knowledge, there are no reports on the usefulness of preoperative arterial embolization. Intraoperative and postoperative bleeding can also lead to airway problems. To avoid unnecessary tracheostomy and ligation of the external carotid artery, preoperative vascular embolism should be considered while removing large tumors or tumors with high blood flow. The current report outlines a case of a 32-year-old woman with a tongue solitary fibrous tumor, who underwent right lingual artery embolization with 300-500 and 500-700 $\mu \mathrm{m}$ embosphere microspheres through a vascular catheter the day before surgical resection. The encapsulated tumor was completely excised under general anesthesia with little to no bleeding during the operation.
\end{abstract}

\section{Introduction}

Solitary fibrous tumors (SFT) are derived from mesenchymal cells originating mainly from the pleura, and cases arising in the head and neck region are rare (especially those that bleed). Many studies reporting tongue SFT treatments exist (1-6), but none have focused on the usefulness of preoperative arterial embolization. According to a recent systemic review of the literature that analyzed 2,629 studies on oral and maxillofacial SFT (7), larger tumors are more likely to be malignant and more aggressive, necessitating a complete excision. For bleeding oral tumors, it is necessary to consider the surgical

Correspondence to: Dr On Hasegawa, Department of Oral and Maxillofacial Surgery, Tokyo Medical University, 6-7-1 Nishishinjyuku, Shinjyuku-ku, Tokyo 160-0023, Japan

E-mail: on-h@tokyo-med.ac.jp

Abbreviation: SFT, solitary fibrous tumor

Key words: solitary fibrous tumor, embolization, tongue, right lingual artery, STAT6 procedure carefully because bleeding during surgery and postoperative care may lead to incomplete resection and airway problems. We treated a 32-year old woman with a gradually increasing and dysarthria of hypervascular tongue SFT by applying preoperative arterial embolization and then removed the tumor safely and completely. At the time of this report, two years have passed without recurrence.

\section{Case report}

A 32-year-old woman without remarkable medical or family histories had been aware of a mass on the lower surface of her tongue that had increased in size for half a year before her first consultation. Her face was bilaterally symmetric without regional lymph node swelling, but we noticed she had dysarthria (probably due to the mass). Her routine laboratory blood work was normal. We found an elastic and hard $30-\mathrm{mm}$ tumor in the right lower part of the tongue. Non-contrast-enhanced computed tomography (CT) images revealed a tumor shadow with a clear border and uniform margins in the right sublingual gap. Dynamic CT images of arterial phase showed a strong enhancement with the inflow from an enlarged right lingual artery thought to be a nutrient vessel. Dynamic CT images of venous phase showed washed out contrast media. Non-contrast-enhanced T1-weighted images showed many point-like low and high signal areas, which is called a salt and pepper appearance. T2-weighted image consisted mainly of high signals compared with spinal cord and was partly accompanied by a cord-like low signal area. An ultrasound showed a pulsatile blood flow signal into the mass. We did not perform a histopathological examination to avoid massive bleeding. We diagnosed the patient with hypervascular tongue tumor, and at this stage, paraganglioma was suspected.

We performed a transarterial embolization for the right lingual artery embolization with 300-500 and 500-700- $\mu \mathrm{m}$ embosphere microspheres (BioSphere Medical) through a vascular catheter the day before the operation. The angiography after the embolization confirmed the disappearance of the dense tumor (Fig. 1). The next day, we completely excised the encapsulated tumor under general anesthesia with little to no bleeding during the operation. The size of the resected specimen after surgery was $31 \times 28 \times 18 \mathrm{~mm}$, which was slightly smaller than the size from preoperative CT findings. In addition, the tumor was wrapped in a thin fibrous capsule with a reddish white and solid appearance. 

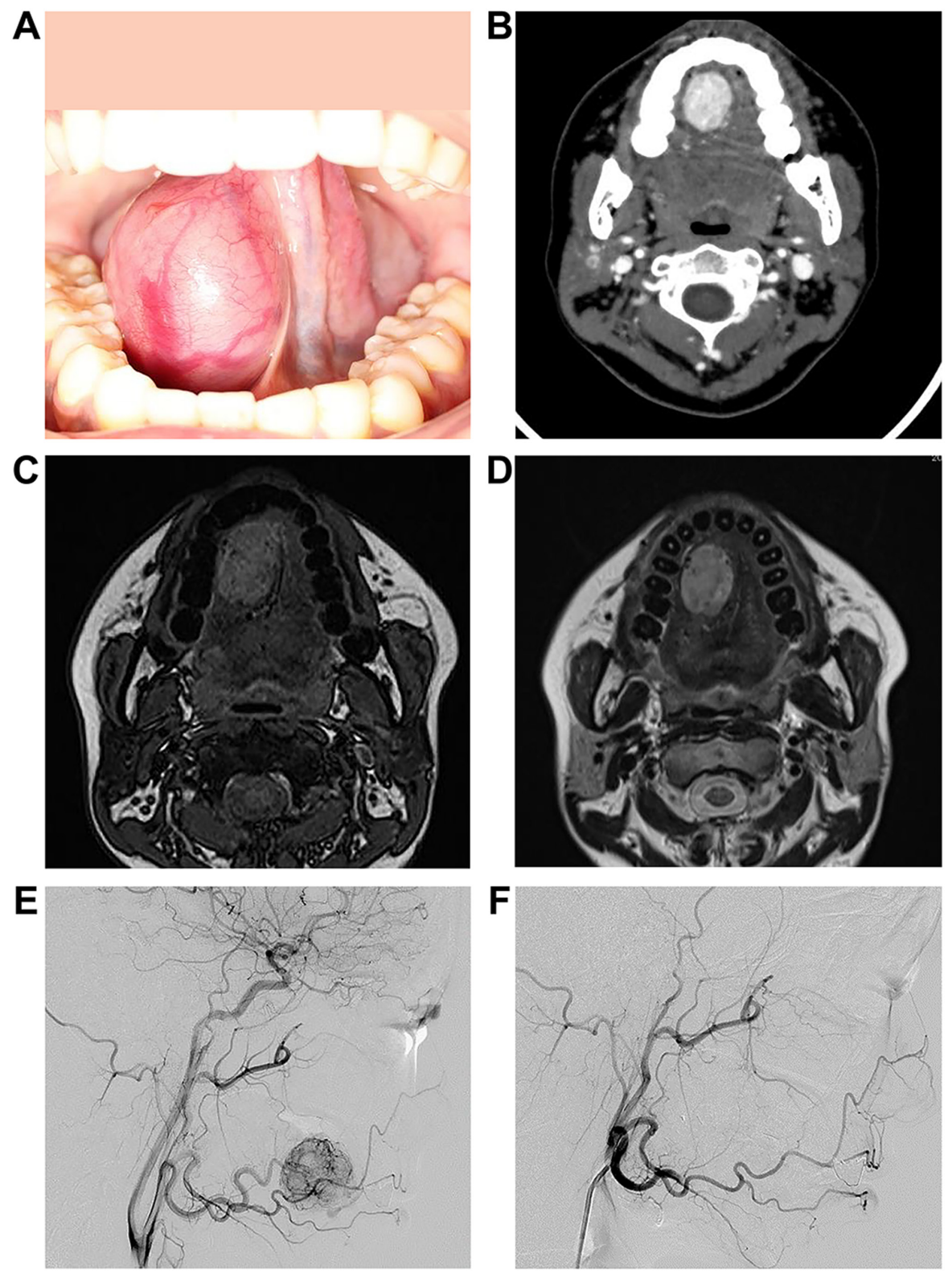

Figure 1. Images captured prior to tumor resection. (A) Intraoral photograph. Elastic and hard tumor in the lower right part of the tongue. (B) Contrast-Enhanced CT: 31×21x27 mm tumor with strong staining in the right sublingual space. (C) MRI T1-weighted image: Salt and pepper appearance. (D) MRI T2-weighted image: Mainly high signal with a cord-like low signal area. ( $\mathrm{E}$ and F) Angiographic findings after right lingual artery embolization. The angiography after embolization confirmed the disappearance of the dense tumor.

Pathology reported disordered growth of the tumor cells with different densities, and an intricate structure and floral arrangement. In addition, the tumor presented a cleft-like blood vessel, and a staghorn-like blood vessel cavity.

Immunohistochemical staining showed spindle cells with CD34-positive diffusivity, and scattered positive STAT6 spindle cells. S-100 protein was negative. The final diagnosis based on findings was tongue SFT (Fig. 2).

\section{Discussion}

SFT was first reported as a pleural tumor in 1931 by Klemperer and Rabin, and since a long time it has been thought to be derived from the pleura (8). According to the 2013 WHO bone/soft tissue tumor classification, the tumor is a moderate malignant tumor and rarely occurs in the oral region (9). Few polycystic SFTs on the tongue have been reported.

Histopathological findings of SFTs show horn-like blood vessels and tumor cells distributed in a 'pattern-less' pattern. According to Chan's diagnostic criteria, CD34 is an effective diagnostic marker (10). Robinson et al (11) described 51 SFTs with NAB2 and STAT6 genes fusions. Thus, we evaluated both markers (CD34 and STAT6) in our patient. Since this case was that of a hypervascular tumor, histopathological examination was not performed before surgery. In the image, paragangliomas indicate the differential diagnosis.

The first choice of treatment is surgical resection, and preoperative embolization is effective for highly irrigated tumors to avoid intraoperative bleeding and incomplete removal, which leads to high local recurrence rates (12). SFT surgery combined with preoperative vascular embolism 
Resected specimen

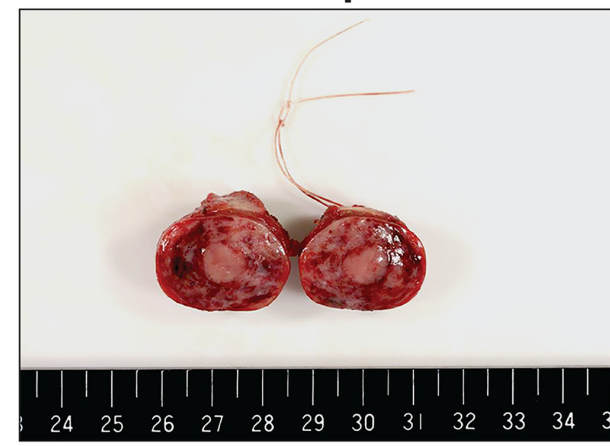

CD34

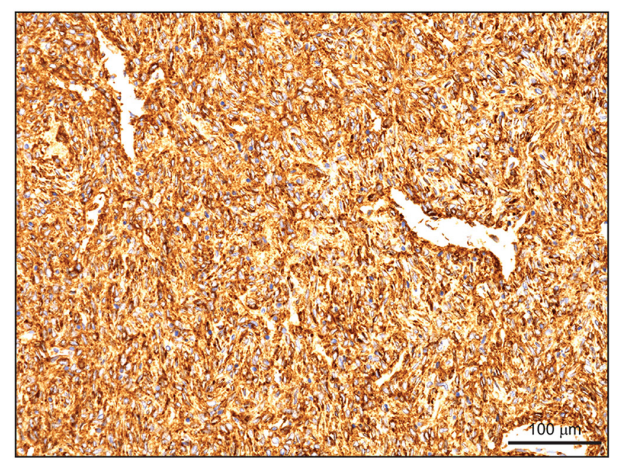

H\&E

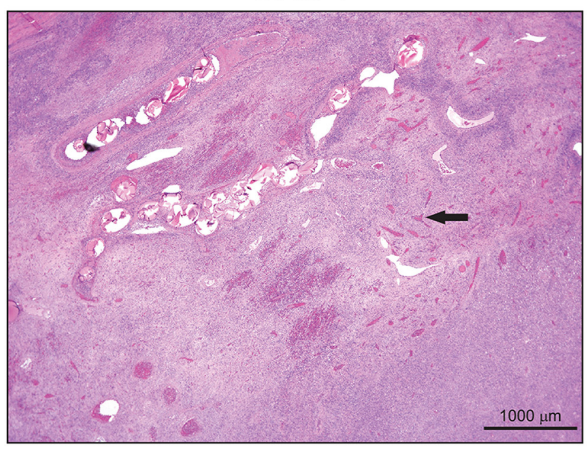

STAT6

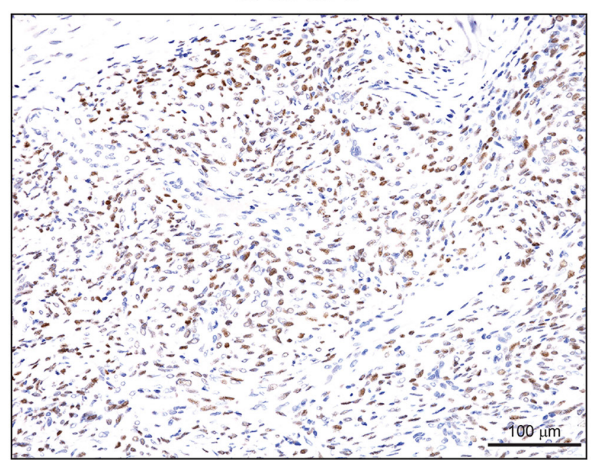

Figure 2. Resected specimen photograph. The tumor was 31x28x18 mm spheroid tumor wrapped in a thin fibrous capsule with a solid reddish white surface. Hematoxylin and eosin (magnification, x20) staining showing pattern-less growth of tumor cells, cleft blood vessels and staghorn-like vessel cavities (black arrow). Immunohistochemical staining revealed positive CD34 (magnification, x200) diffuse cells, and positive STAT6 (magnification, x200) scattered spindle cells. CD, cluster of differentiation.

has already been reported (13-16), but not in the tongue. Intraoperative and postoperative bleeding can also lead to airway problems. To avoid unnecessary tracheostomy and ligation of the maxillary artery, preoperative vascular embolism may be considered aggressively when removing large tumors or tumors with high blood flow. In the case of our patient, we embolized the right lingual artery on the day before the resection, and were able to excise the encapsulated tumor successfully under general anesthesia the next day with little to no bleeding.

Many SFTs are benign and have a relatively good prognosis. However, this tongue tumor is considered to be of intermediate malignancy, and Fletcher et al (9) have reported a 10\% recurrence rate. In some cases, recurrences or metastases have been reported after more than 10 years, and follow-ups are required for at least the first two years after the resection (17). In addition to regular contrast-enhanced CT examinations, we will consider abdominal ultrasonography and chest radiographs. At the time of this report, 2 years have passed and the patient has followed a good course without recurrence. We plan for a long-term follow-up.

We treated a patient with tongue SFT by applying preoperative arterial embolization, and then removed the tumor safely and completely. Transarterial embolization should be actively combined with surgery for safe and reliable removal of hypervascular oral tumors in the oral cavity.

\section{Acknowledgements}

The authors would like to thank Dr Toshitaka Nagao and Dr Ai Enomoto of the Department of Anatomic Pathology, Tokyo Medical University professional opinion about pathological discussion and Dr Kazuhiro Saito of the Department of Radiology, Tokyo Medical University for excellent management of the embolization procedure.

\section{Funding}

No funding was received.

\section{Availability of data and materials}

All data generated or analyzed during this study are included in this published article.

\section{Authors' contributions}

$\mathrm{OH}$ acquired patient data, performed the literature review and edited the manuscript. DC conceived and designed the present study. MW and MK acquired the data, provided clinical advice and revised the manuscript. DY gave radiological advice. $\mathrm{OH}$ wrote the manuscript. All authors read and approved the final version of the manuscript.

\section{Ethics approval and consent to participate}

Not applicable.

\section{Patient consent for publication}

A signed written informed consent was obtained from the patient. 


\section{Competing interests}

The authors declare that they have no competing interests.

\section{References}

1. Piatelli A, Fioroni M and Rubini C: Solitary fibrous tumor of the tongue. Oral Oncol 34: 431-434, 1998.

2. Yamashita Y, Satoh T and Goto M: Solitary fibrous tumor of the tongue: A case report with immunohistochemical studies. Int J Oral Maxillofac Surg 31: 681-683, 2002.

3. Vafiadou M, Dimitrakopoulos I, Georgitzikis I, Hytiroglou P, Bobos M and Karakasis D: Solitary fibrous tumor of the tongue: Case report and literature review. Int J Oral Maxillofac Surg 37: 1067-1069, 2008.

4. Migita M, Yoshino M, Kobayashi D, Shiomi S, Enatsu K, Shigematsu S and Ohata H: A large solitary fibrous tumor of the tongue. J Oral Maxillofac Surg 70: 871-874, 2012.

5. Shnayder Y, Greenfield BJ, Oweity T and DeLacure MD: Malignant solitary fibrous tumor of the tongue. Am J Otolaryngol 24: 246-249, 2003.

6. Kaneko T, Kawano R, Horie N and Shimoyama T: Solitary fibrous tumour of the tongue: A case report. Oral Surg 11: 65-68, 2018.

7. De Morais EF, Martins HD, Rodrigues KS, de Franca GM, da Silveira ÉJ and Freitas RA: Clinicopathologic analysis of ora and maxillofacial solitary fibrous tumor. Am J Clin Pathol 154: 15-22, 2020.

8. Klempere P and Rabin CB: Primary neoplasm of the pleura: A report of 5 cases. Arch Pathol 11: 385-412, 1931.

9. Fletcher CDM, Bridge JA and Lee JC: Extrapleural solitary fibrous tumor. In: WHO classification of tumors of soft tissue and bone. Fletcher CD, Bridge JA, Hogendoorn PC and Mertens F (eds). IARC Press, Lyon, pp80-82, 2013.
10. Chan JK: Solitary fibrous tumor-everywhere, and a diagnosis in vogue. Histopathology 31: 568-576, 1997.

11. Robinson DR, Wu YM, Kalyana-Sundaram S, Cao X, Lonigro RJ, Sung YS, Chen CL, Zhang L, Wang R, Su F, et al: Identification of recurrent NAB2-STAT6 gene fusions in solitary fibrous tumor by integrative sequencing. Nat Genet 45: 180-185, 2013.

12. Craven JP, Quigley TM, Bolen JW and Raker EJ: Current management and clinical outcome of hemangiopericytoma. Am J Surg 163: 490-493, 1992.

13. Weiss B and Horton DA: Preoperative embolization of a massive solitary fibrous tumor of the pleura. Ann Thorac Surg 73: 983-985, 2002.

14. Zeitler DM, Kanowitz SJ and Har-El G: Malignant solitary fibrous tumor of the nasal cavity. Skull Base 17: 239-246, 2007.

15. Yokoyama Y, Hata K and Kanazawa T: Giant solitary fibrous tumor of the pelvis successfully treated with preoperative embolization and surgical resection: A case report. World J Surg Oncol 13: 164-167, 2015.

16. Yammine K, Nasser HA, Hadi U, Natout MA, Najjar V and Tayar C: Salvage preoperative embolization of an infratemporal solitary fibrous tumor: A case report with review of the literature. Medicine (Baltimore) 97: e0251, 2018.

17. Daigeler A, Lehnhardt M, Langer S, Steinstraesser L, Steinau HU, Mentzel T and Kuhnen C: Clinicopathological findings in a case series of extrathoracic solitary fibrous tumors of soft tissues. BMC Surg 6: 10, 2006.

() $(-)$ This work is licensed under a Creative Commons Attribution-NonCommercial-NoDerivatives 4.0 International (CC BY-NC-ND 4.0) License. 